\title{
DESAFIOS E VANTAGENS DA UTILIZAÇÃO DE CRITÉRIOS PROBABILÍSTICOS NO PLANEJAMENTO DE SISTEMAS ELÉTRICOS
}

\author{
João Marcos Lima* \\ jm. lima@copel.com
}

\author{
Elizete Maria Lourenço ${ }^{\dagger}$ \\ elizetedeletrica.ufpr.br
}

\author{
Marcus Theodor Schilling $\ddagger$ \\ schilling@ic.uff.br \\ *Companhia Paranaense de Energia - COPEL \\ Rua José Izidoro Biazetto, 158 - Boco A - Sala 268 \\ CEP 81200-240 - Curitiba - PR \\ ${ }^{\dagger}$ Universidade Federal do Paraná - UFPR \\ Departamento de Engenharia Elétrica - Setor de Tecnologia \\ C.P. 19011 - CEP 81531-990 - Curitiba - PR \\ $\ddagger$ Universidade Federal Fluminense - UFF \\ Departamento de Engenharia Elétrica - Instituto de Computação \\ Rua Passo da Pátria 156, Bloco E, sala 350 \\ São Domingos- Ingá - CEP 24210-240 - Niterói - RJ
}

\begin{abstract}
Challenges and Benefits of the Use of Probabilistic Criteria in Electric Power Systems Planning

The Brazilian power grid has been traditionally designed and operated using a set of deterministic reliability criteria. This method has played an important role in the design and operation of the Brazilian bulk power system for decades. However, deterministic criterions tend to use available resources less optimally and stress facilities in the context of transmissions capacity. In the expansion planning, for instance, the main deterministic criterion is the well know $n-1$. With regard to the approval of scheduled maintenance of transmission equipment on the basic grid, the Brazilian Independent System Operator (ISO) is the $n-2$. This criterion requires that the electrical system support the unavailability of any particular transmission element that has been deactivated for maintenance purposes, as well as any other contingency situations
\end{abstract}

Artigo submetido em 26/11/2010 (Id.: 1220)

Revisado em 14/01/2011, 08/08/2011, 28/10/2011

Aceito sob recomendação do Editor Associado Prof. Arturo Bretas that may arise, without violating any system constraints. This paper discusses the limitations of the deterministic reliability criteria and proposes a new approach entirely based on probabilistic techniques. The new method allows a more efficient scheduled maintenance during medium load workdays, without significantly increasing the risk level of the system. The expected result of implementing this new criterion is a reduction in operations and maintenance costs, which should in turn reduce the electricity rates paid by end users.

KEYWORDS: Reliability, deterministic criteria, probabilistic criteria, severity index.

\section{RESUMO}

O sistema elétrico brasileiro tem sido projetado e operado através do uso de um conjunto de critérios de confiabilidade determinísticos. Esses critérios têm cumprido um papel importante no desenho e na operação do sistema durante décadas. Entretanto, tendem à utilização menos otimizada dos recursos disponíveis e no excesso de instalações de reserva 
de capacidade de transmissão, com impacto direto nos custos associados ao planejamento e operação do sistema. No planejamento da expansão, por exemplo, o critério determinístico mais utilizado é o $n$-1. Com relação à aprovação de intervenções programadas na rede básica, o principal critério determinístico adotado pelo Operador Nacional do Sistema Elétrico (ONS) é o $n$-2. Esse critério estabelece que o sistema elétrico deve suportar a indisponibilidade simultânea de um elemento de transmissão que esteja fora de operação para manutenção e de qualquer outro elemento por contingência, sem que ocorram violações dos indicadores de desempenho do sistema. Este artigo discute as limitações desses critérios determinísticos e propõe uma nova metodologia para avaliação de intervenções programadas, com base em técnicas probabilísticas. A nova metodologia permite uma avaliação mais precisa e flexível de agendamento de interrupções durante dias úteis em período de carga média, sem que o grau de risco do sistema se eleve a níveis preocupantes. Os resultados esperados com esse novo critério correspondem à redução dos custos de operação e de manutenção que, por sua vez, implicam na redução das tarifas de energia paga pelos consumidores.

PALAVRAS-CHAVE: Confiabilidade, critérios determinísticos, critérios probabilísticos, índice severidade.

\section{INTRODUÇÃO}

O planejamento da operação de curto prazo do sistema elétrico brasileiro tem sido realizado basicamente através de critérios determinísticos. O conhecido critério determinístico $n$ 1 é também amplamente utilizado no planejamento da expansão. No entanto, para o planejamento da operação, ao qual estão relacionadas as intervenções na rede básica, o principal critério adotado pelo ONS é o $n-2$. Este critério considera que, estando um dos elementos do sistema indisponível, sobretudo para manutenção programada, o sistema deverá suportar a contingência de qualquer outro elemento, sem que ocorram violações de níveis de tensão e carregamento, restrições de atendimento à carga e, de preferência, que não sejam alteradas as programações energéticas entre áreas.

O critério $n$-2 não é o único critério adotado pelo ONS no planejamento da operação. Dependendo das circunstâncias, esse critério pode ser elevado ao nível 3, ou n-3. Além disso, a ocorrência de apagões, como o ocorrido no dia 10 de novembro de 2009, reforça a adoção de uma postura mais conservadora pelo ONS em relação à operação do sistema, que tende a restringir ainda mais a liberação de equipamentos para a manutenção programada.

Limitações decorrentes do uso exclusivo de critérios determinísticos podem ser evitadas ou pelo menos minimizadas por meio da utilização de critérios probabilísticos, uma vez que a literatura na área reconhece a natureza estocástica dos sistemas elétricos (Billinton et al., 2001; Lima, 2007; Schilling et al., 1995; Schilling et al., 1998; Schilling et al., 2009).

Adicionalmente, constata-se uma tendência na literatura em se calcular os níveis de risco utilizando técnicas probabilísticas em diferentes níveis de agregação: por área, por nível de tensão, por centro de carga, por empresa, global. Essa tendência tem sido incentivada em função de fatores como reconhecimento da natureza inerentemente probabilística dos sistemas de potência; tentativas para evitar desperdícios oriundos de decisões puramente determinísticas e, principalmente, escassez de recursos financeiros que obrigam a investigação mais minuciosa do comportamento do sistema, levando-se em conta riscos versus custos operacionais associados (Schilling et al., 1998).

Cabe ressaltar, também, que no âmbito do planejamento da operação, barreiras que por um longo tempo limitaram a utilização das técnicas probabilísticas já estão superadas, como a limitação tecnológica das ferramentas computacionais e matemáticas. Apesar disso, no Brasil, a utilização de análises probabilísticas no planejamento da operação ainda não atingiu um nível satisfatório para tomada de decisão.

Nesse sentido, este trabalho propõe uma nova metodologia baseada em técnicas probabilísticas que buscam conciliar os objetivos conflitantes que envolvem a questão da segurança do sistema e o desligamento de equipamentos para realização de manutenção programada.

Nessa nova abordagem, problemas relacionados à ocorrência de apagões, por exemplo, deixam de ser determinantes no que se refere aos desligamentos programados, já que as causas de apagões estão muito mais relacionadas ao despacho econômico e a eventos catastróficos do que com as intervenções programadas. As medidas adotadas pelo ONS com relação ao apagão de 2009 propõe, entre outras medidas, a ampliação da produção de energia térmica para reduzir a dependência do sistema com relação a Itaipu e, consequentemente, melhorar a segurança do sistema. Esse procedimento reforça a relação significativa entre apagões e despacho econômico mencionada, ressaltando a relevância da metodologia probabilística proposta neste trabalho.

Finalmente, verifica-se que a falta de conhecimento com relação às potencialidades das técnicas probabilísticas, a dificuldade de interpretação de seus resultados e a dificuldade de obtenção de dados estatísticos são os principais obstáculos para uma maior difusão dessas técnicas no planejamento da operação. Assim, este trabalho tem também por objetivo encorajar a difusão e o uso de metodologias probabilísticas como ferramenta de suporte à decisão no planejamento da operação de sistemas elétricos, especialmente aquelas relacionadas à aprovação de manuntenção programada. 


\section{CRITÉRIOS DETERMINÍSTICOS}

\subsection{Metodologia Determinística Adotada Atualmente}

A Figura 1 apresenta, de forma simplificada, a metodologia operacional $n-2$, adotada atualmente pelo ONS para análise e aprovação de desligamentos programados. Se uma companhia de transmissão necessita desconectar algum equipamento da Rede Básica (a qual engloba apenas os elementos em tensões de $230 \mathrm{kV}$ ou superiores) para manutenção, tais como um transformador ou uma linha de transmissão, é adotado o procedimento apresentado na Figura 1.

Esta análise leva em conta o patamar de carga, o cenário de intercâmbio entre áreas elétricas, os limites nominais dos equipamentos, bem como outros desligamentos já aprovados (vide bloco 1, Figura 1). Todas essas condições definem o caso de referência, também denominado caso base.

O equipamento solicitado é, então, removido do caso base. Se sua saída implica em violações das restrições de operação, carga ou segurança, tais como quedas de tensão ou sobrecarga, determinadas ações são verificadas (vide bloco 3). Essas ações podem incluir, por exemplo, redespacho de geração e mudanças no controle de tensão.

Se as violações não podem ser contornadas (bloco 4), o pedido de manutenção é rejeitado (bloco 5). Caso contrário, se as medidas operativas obtiverem sucesso ou se a saída do equipamento não causar qualquer violação no sistema, um conjunto pré-definido de contingências simples são analisadas (bloco 6), considerando-se agora os limites de carregamento de emergência. Nesta situação, o sistema já se encontra na condição n-2, pois um equipamento está fora de operação para manutenção juntamente com a contingência simples em análise.

O processo descrito nos blocos 2-4 é repetido nos blocos 7-9. Se não houver violações depois da análise $n$-2, o pedido de manutenção é aprovado (bloco 10); caso contrário, é rejeitado.

Este procedimento é claramente determinístico. Ignora a natureza estocástica do sistema elétrico, uma vez que todas as contingências são consideradas com a mesma probabilidade de ocorrência. Portanto, não permite flexibilidade no conjunto de critérios exigidos para pedidos de agendamento de manutenção de equipamentos. Relativamente à contingência, não há distinção quanto à extensão da linha de transmissão, nem quanto ao nível de tensão ou quanto ao tipo de equipamento (linha de transmissão, transformador, disjuntor, etc.). Consequentemente, a grande maioria dos pedidos de manutenção é aprovada apenas para o patamar de carga leve, ou

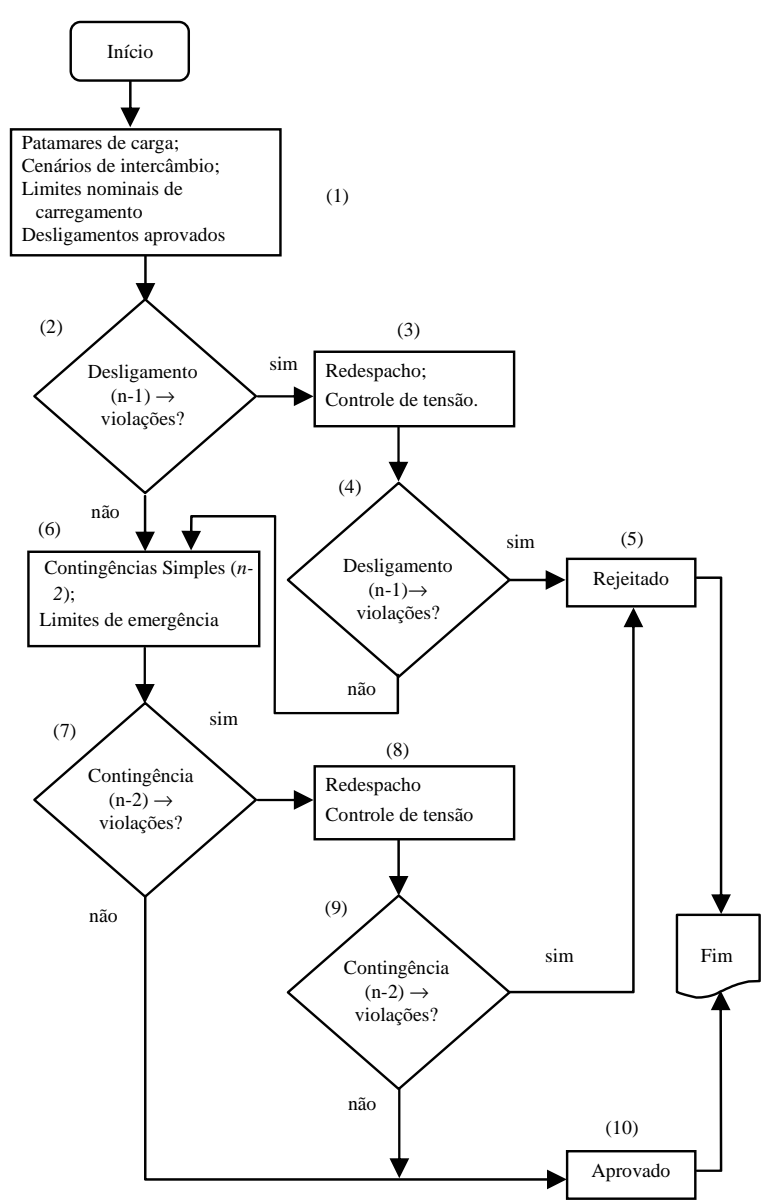

Figura 1: Atual critério determinístico $n-2$ adotado pelo ONS.

seja, em domingos e feriados e durante o período noturno, resultando em maiores custos e riscos.

\subsection{Limitações dos Métodos Determinís- ticos}

Esta seção apresenta dois pequenos exemplos com o objetivo de ilustrar as limitações dos critérios determinísticos. O primeiro exemplo, associado à Figura 2, refere-se à utilização de critério determinístico no planejamento da expansão. Já o segundo exemplo (Figura 3), refere-se à utilização desse método no planejamento da operação, tais como os adotados atualmente pelo ONS e descritos na Seção anterior.

Considere o sistema elétrico de atendimento à subestação $\mathrm{B}$ ilustrado na Figura 2. Neste caso, a subestação B atende a carga $\mathrm{C}$ através do transformador TR1 e está conectada à subestação A através da linha de transmissão LT1. O planejamento da expansão, baseado no critério $n$-1, estabelece 
a construção da linha de transmissão LT2 e a instalação do transformador TR2 como forma de garantir o atendimento da carga $\mathrm{C}$ quando da ocorrência de contingências simples.

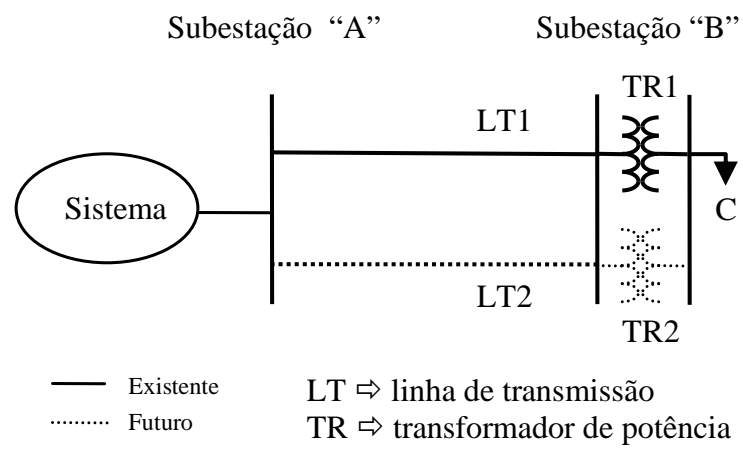

Figura 2: Conexão da subestação $B$ ao sistema elétrico através de elementos em série.

Uma das limitações do critério $n$-1 refere-se à entrada das expansões previstas, representadas por LT2 e TR2 na Figura 2. Ou seja, o critério n-1 não é capaz de definir a prioridade dessas instalações no caso em que os recursos disponíveis não sejam suficientes para implementar a entrada simultânea desses elementos. Isto deve-se ao fato de que a saída de qualquer um deles interrompe o atendimento à carga $\mathrm{C}$.

Embora o exemplo ilustrado pela Figura 2 seja muito simples, ele consiste no dilema que afeta quase todas as distribuidoras que decidem pela expansão de seus sistemas radiais de tensão inferior a $230 \mathrm{kV}$, conectados à Rede Básica através de uma única linha ou de uma única fonte.

Estas questões estão claramente associadas à natureza determinística do critério $n$-1, que impossibilita a comparação do nível de risco que cada desligamento oferece ao sistema.

A Figura 3 ilustra o uso do critério determinístico no planejamento da operação. Neste caso, a subestação B, que atende a carga $C$ através dos transformadores TR1 e TR2, está conectada à subestação A através das linhas de transmissão LT1 e LT2. Caso seja necessário o desligamento de uma das linhas ou de um dos transformadores para manutenção - considerando que: a) tanto a linha como o transformador remanescente têm capacidade de atender a carga C; e $b$ ) tal desligamento não implica em violação dos níveis de tensão de acordo com o critério $n-2$; - o resultado da aplicação do método determinístico apresentado na Seção 2.1 indicará que o desligamento solicitado só pode ser liberado para o menor nível da carga $\mathrm{C}$, que normalmente ocorre aos domingos, feriados e durante as madrugadas.

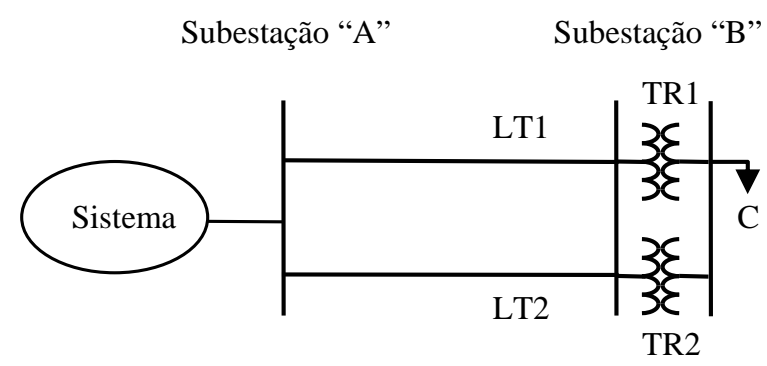

Figura 3: Conexão da subestação $B$ ao sistema elétrico através de elementos em paralelo.

Esses fatores aumentam as chances de acidentes ou ocorrências indesejáveis, principalmente quando os desligamentos ocorrem no período noturno. Além disso, constata-se aumento dos custos da manutenção, sobretudo devido ao maiores custos associados à contratação de mão de obra.

Ao contrário dos métodos determinísticos, a utilização de técnicas probabilísticas permite associar às análises acima os custo de não faturamento, de interrupção, as perdas econômicas e sociais devido à probabilidade de interrupção da carga, entre outros, possibilitanto a utilização mais racional dos recursos disponíveis (Lima, 2007), conforme discutido nas próximas seções.

\section{FERRAMENTAS E MÉTODOS}

A seguir são apresentados tanto o modelo computacional $\mathrm{NH} 2$, como o índice de severidade, ambos utilizados pela metodologia probabilística proposta na Seção 4.

\subsection{O programa NH2}

O NH2 é um programa comercial desenvolvido pelo Centro de Pesquisas em Energia Elétrica - Cepel. O programa $\mathrm{NH} 2$ permite realizar análises de confiabilidade composta de geração e transmissão de sistemas elétricos de grande porte. Permite, também, realizar outras avaliações determinísticas e probabilísticas. Suas principais características são:

- Modelagem não linear da rede elétrica;

- Ambiente de solução de sistemas de potência que compreende:

- Solução de fluxo de potência pelo método Newton-Raphson; 
- Solução de fluxo de potência ótimo não linear, baseado no método de pontos interiores, com minimização do corte de carga;

- Análises de contingências simples;

- Análise de lista de contingências (abordagem determinística);

- Análises de lista de contingências (abordagem determinística);

- Fluxo de potência probabilístico.

- Fluxo de potência ótimo usado para adoção de medidas corretivas;

- Comportamento aleatório (randômico) de unidades geradoras e elementos de transmissão que são representados por modelos markovianos de dois ou múltiplos estados;

- Modo de falha comum;

- Análise de confiabilidade probabilística pelo método de enumeração de estados ou Simulação Monte Carlo;

- Índices tradicionais de frequência e duração, estratificados em três níveis: sistema, área e barras;

- Estatística adicional: função densidade de probabilidade das variáveis selecionadas (fluxos, tensão, intercâmbio), índices de modo de falha, etc.

O NH2 é usado por mais de trinta empresas do setor elétrico brasileiro, incluindo o ONS e a Empresa de Pesquisa Energética - EPE. Esse programa tem sido utilizado pelo grupo de planejamento do sistema interligado nacional desde a sua primeira versão. Nos últimos quatro anos, o ONS tem utilizado o programa $\mathrm{NH} 2$ para o diagnóstico do desempenho do sistema interligado nacional por meio de análises de confiabilidade probabilística, considerando o horizonte de médio prazo (três anos) do Plano de Ampliações e Reforços - PAR.

O programa NH2 permite a seleção de um dos dois métodos para avaliações de confiabilidade composta: $a$ ) método de enumeração de estados ou $b$ ) simulação Monte Carlo. Na utilização do método de enumeração de estados ou contingências, essas são selecionadas a partir de uma lista pré-definida, que pode abranger todo o sistema ou apenas uma pequena área. Combinando os elementos da lista, diferentes níveis de contingências podem ser gerados, desde contingências simples (apenas um elemento em falha e os demais em operação) até o enésimo nível. Como esse procedimento pode resultar em um combinação elevada de estados, um dos seguintes critérios de parada pode ser utilizado (combinado ou isolado):

- Nível de contingência: o processo avalia somente níveis pré-determinados, ou seja, simples, duplas ou múltiplas.
- Probabilidade: o processo avalia somente contingências com probabilidade superior a um valor pré-definido.

O método de enumeração de estados é normalmente mais fácil de se entender e implementar. É normalmente muito eficiente para contingências simples ou quando o espaço de estados é relativamente pequeno. Entretanto, o esforço computacional tende a crescer rapidamente para níveis mais elevados de contingências ou quando a probabilidade das contingências é alta.

Os índices calculados pelo método de enumeração apresentam normalmente limites inferiores aos valores reais, uma vez que a enumeração de todos os estados possíveis de contingências em um sistema de potência real é impraticável. Ou seja, os índices reais nunca serão inferiores aos índices calculados. Portanto, quanto maior o nível de contingências processado, mais próximos dos valores reais estarão os indíces obtidos. O método de enumeração de estados, quando limitado ao primeiro nível (contingências simples), é muito similar ao critério $n-1$.

A simulação Monte Carlo, por sua vez, seleciona um estado do sistema baseado na amostra da função de distribuição de probabilidade conjunta. Se os componentes são estatísticamente independentes, os estados do sistema são selecionados a partir das funções de distribuição de probabilidade de cada componente. O método é muito versátil, uma vez que permite utilizar diferentes tipos de distribuição de probabilidade e permite representar o comportamento aleatório da carga facilmente.

Diferente da enumeração de estados, os resultados obtidos pela simulação Monte Carlo não representam os limites inferiores, mas estimativas do desempenho real do sistema. $\mathrm{O}$ esforço computacional não depende do tamanho ou complexidade do sistema. Entretanto, é muito afetado pela precisão exigida. Para contornar esse problema, técnicas de redução da variância podem ser usadas, como regressão generalizada ou importância da amostra.

A adequação de cada caso é determinada com o objetivo de se identificar violações em componentes do sistema. As violações podem ser eliminadas por meio de métodos de otimização.

As análises de adequação podem ser realizadas pelo método linear ou não linear, tanto para o fluxo de potência convencional como para o fluxo de potência ótimo.

O diagnóstico do desempenho do sistema é realizado através da análise dos índices de confiabilidade probabilística e também por informações adicionais, tais como identificação e classificação dos casos mais severos, função de distribuição de probabilidade das variáveis selecionadas e violações 
estatísticas (Cepel, 2005; EPRI, 1987; Mello et al., 1991; Rei et al., 2006).

\subsection{0 índice severidade}

O índice severidade (Schneider et al., 1989) é um dos mais importantes indicadores de risco probabilístico. Este é expresso em termos de minutos sistema, minutos padrão ou, simplesmente, minuto. Um minuto sistema representa a energia interrompida equivalente à ponta de carga do sistema durante um minuto. $\mathrm{O}$ índice severidade é calculado através da divisão do valor estimado de energia interrompida (MWh) pela base de potência (MW) (que corresponde à ponta de carga do sistema, área, região, barra, etc). Este valor numérico é multiplicado por 60 para convertê-lo em minutos sistema). O termo minutos sistema será indicado simplesmente por minutos daqui para frente.

Pelo fato de ser um indicador normalizado, o índice de severidade permite a comparação de sistemas de portes e naturezas distintas, advindo daí a sua importância. É também um indicador bastante flexível, pois pode ser avaliado para diferentes agregações espaciais e temporais. Matematicamente, a severidade (S) é expressa por (Schneider et al., 1989):

$$
S=\frac{E E N S}{P S} \cdot 60
$$

onde $S$ é a severidade (em minutos), $P S$ é a ponta de carga (em MW) e $E E N S$ é a expectativa de energia não suprida (em MW.h). A EENS é definida por (GCPS and SGC, 1990):

$$
E E N S=\sum_{i \in \Delta} f_{i} d_{i} c_{i}
$$

A Tabela 1 mostra a classificação da confiabilidade do sistema elétrico brasileiro para vários níveis de severidade, adotada pelo ONS (ONS, 2004).

A severidade é um dos poucos indicadores probabilísticos de curso internacional que já dispõe de uma escala de valoração classificatória, com base logarítmica. O conceito que o embasa é o da classificação de eventos de forma semelhante ao empregado no tratamento de terremotos, onde a escala é diferenciada da antecedente por uma ordem de grandeza.

\section{CRITÉRIO PROBABILÍSTICO PARA ANÁLISES DE INTERVENÇÕES PRO- GRAMADAS NA REDE BÁSICA}

A utilização de métodos probabilísticos em sistemas elétricos de potência tem evoluído lentamente desde os anos sessenta. Isto é evidente a partir da literatura existente (ONS, 2006; Schneider et al., 1989; Ran, 2003; Soares, 2002), que mostra algumas aplicações interessantes, tanto no planejamento como na operação desses sistemas.

A exemplo dessas referências, a metodologia probabilística adotada aqui é baseada nas seguintes premissas: (i) a análise de indisponibilidade do planejamento da operação deve considerar limites normais de operação para as análises que se referem aos desligamentos programados e limites de curta duração para os níveis de tensão e carregamento em situações de emergência, e (ii) a análise de indisponibilidade do planejamento da operação não deve considerar falhas em elementos de transmissão que atendem consumidores e subestações da Rede Básica; (iii) o caso base utilizado para a análise de confiabilidade deve refletir o cenário de intercâmbio real ou esperado.

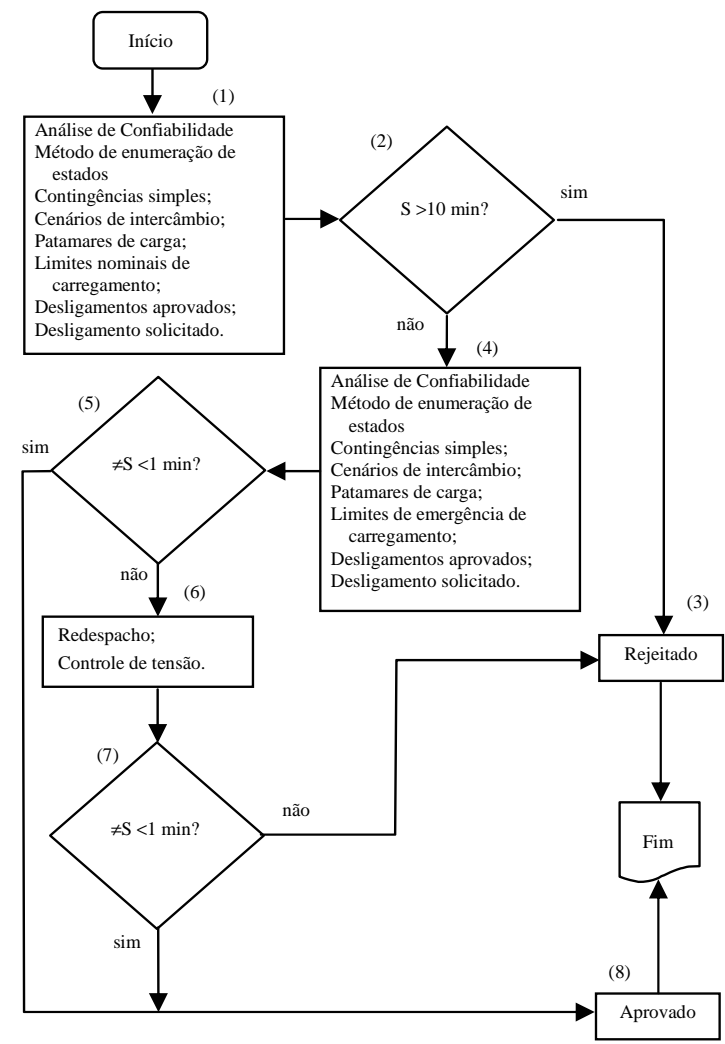

Figura 4: Metodologia para Avaliação do Nível de Risco Probabilístico no Planejamento da Operação 
Tabela 1: Classificação do desempenho por severidade (ONS, 2004).

\begin{tabular}{|c|c|c|c|}
\hline Classificação & $\begin{array}{c}\text { Severidade "S" } \\
\text { (sistema-minuto) }\end{array}$ & Interpretação & Comentário \\
\hline Grau 0 & $S<1$ & Favorável & Condição operativa de baixíssimo risco \\
\hline Grau 1 & $1 \leq S<10$ & Satisfatório & Condição operativa de baixo risco \\
\hline Grau 2 & $10 \leq S<100$ & Limítrofe & Condição operativa de risco médio \\
\hline Grau 3 & $100 \leq S<1000$ & Grave & Sério impacto para vários agentes/consumidores \\
\hline Grau 4 & $1000 \leq S$ & Muito Grave & Grande impacto para muitos agentes/consumidores \\
\hline
\end{tabular}

Uma vez consideradas essas três hipóteses, o método proposto para avaliar o nível de risco probabilístico para o planejamento da operação é descrito pela Figura 4. Os passos na sequência de 1 a 8 , facilmente identificados no diagrama, são discutidos a seguir.

Passo 1: A partir das considerações anteriores, que são equivalentes àquelas descritas para a formação do caso base no critério determinístico, realiza-se uma análise de confiabilidade de transmissão pelo método de enumeração de estados. Esta análise deve levar em consideração os desligamentos aprovados, os desligamentos solicitados, a análise de contingência simples para cada nível de carga (pesada, média e leve), os limites de nível de tensão e o carregamento para condições normais de operação. Além disso, deve permitir o controle de taps dos transformadores e a desabilitação do redespacho de geração (bloco 1, Figura 4). A ferramenta computacional utilizada é o NH2, descrito na Seção 3.1. Informações adicionais e detalhes sobre como este tipo de análise é realizada podem ser obtidas em (Lima, 2007)

Passo 2: Se o índice de severidade do sistema para o desligamento solicitado for maior do que 10 minutos (bloco 2, Figura 4), este será rejeitado (bloco 3, Figura 4). O conceito do índice de severidade, definido em (ONS, 2004; Schneider et al., 1989) é brevemente descrito na Seção 3.2;

Passo 3: Se o índice de severidade do sistema for igual ou inferior a 10 minutos, uma nova análise de confiabilidade padrão deve ser processada, considerando agora os limites de emergência para os níveis de tensão e carregamento (bloco 4, Figura 4);

Passo 4: Se a diferença entre os índices do caso atual e do caso base for inferior a 1 minuto, o desligamento é apontado como de baixíssimo risco e, portanto, deve ser aprovado (bloco 8, Figura 4);

Passo 5: Se a diferença entre esses índices for maior do que 1 minuto (bloco 5, Figura 4), algumas ações operativas devem ser avaliadas considerando as contingências mais críti- cas. Estas ações podem incluir redespacho e alterações no controle de tensão (bloco 6, Figura 4);

Passo 6: A análise de confiabilidade de transmissão é realizada novamente considerando-se as novas condições. Se a diferença é maior do que um minuto (bloco 7, Figura 4), o pedido de desligamento para manutenção deve ser rejeitado (bloco 3, Figura 4). Caso contrário, será aprovado (bloco. 8, Figura 4);

Passo 7: Uma vez que o procedimento descrito nos passos 1-6 é concluído e o ONS aprova o desligamento solicitado, esse desligamento deve ser automaticamente incluído no caso base. Análises de desligamentos subsequentes irão, portanto, levar em consideração os desligamentos já aprovados.

O principal objetivo da metodologia descrita acima é não violar o grau de risco do sistema atual quando um ou mais equipamentos são desligados para manutenção (vide Tabela 1). Tipicamente, o risco médio para a Rede Básica durante condições normais de operação devem corresponder ao grau 1 (i.e., 1 minuto $\leq$ Severidade $<10$ minutos). Portanto, se a intenção é preservar o nível de risco do sistema, o limite máximo para um desligamento permitido é de 10 minutos. (bloco 3, Figura 4). Este critério pode ser convenientemente ajustado sempre que desejado.

Além disso, cada desligamento não pode aumentar significativamente o nível de risco do sistema quando limites de emergência são considerados para os níveis de tensão e carregamento. De acordo com o bloco 5, Figura 4, a máxima tolerância adotada nesse caso é de 1 minuto.

Portanto, esta metodologia incorpora o conceito de risco admissível para o sistema (Schneider et al., 1989; Negreiros et al., 2007; Soares, 2002), o que pode ser visto como a principal diferença entre esta e a metodologia determinística usada atualmente e descrita na Seção 2 (Figura 1). 


\section{SIMULAÇÕES E RESULTADOS}

Nesta seção são apresentados os resultados referentes aos pedidos de desligamento de equipamentos em um sistema real, cujas características e simulações são descritas nas seções 5.2 e 5.3. Inicialmente, são apresentados os resultados referentes à aplicação da metodologia probabilística proposta neste trabalho, que combina a utilização do índice de severidade e a avaliação da confiabilidade usando o programa $\mathrm{NH} 2$, conforme descrito na Seção 3. Na sequência, são apresentados os resultados obtidos com a aplicação do critério determinístico adotado atualmente pelo ONS e descrito na Seção 2.

\subsection{Sistema Teste}

O sistema teste considerado neste estudo compreende o sistema de transmissão que atende o estado do Paraná, tendo por base o ano 2007. O sistema é composto pelo seguinte conjunto de elementos:

- 56 LTs de $230 \mathrm{kV}(4.046 \mathrm{~km})$.

- 19 LTs de $500 \mathrm{kV}(2.560 \mathrm{~km})$.

- 10 TRs de 525/230 kV (7.700 MVA).

- 41 TRs de 230/69 and 230/138 kV (6.600 MVA).

- 8 principais hidroelétricas (30 unidades geradoras): 7,2 GW de potência instalada;

- Ponta de carga do sistema: $4 \mathrm{GW}$;

- Capacidade de intercâmbio: $5 \mathrm{GW}$ entre os subsistemas Sul e Sudeste, em ambos os sentidos.

É importante mencionar que as análises de fluxo de potência envolvidas no processo são realizadas considerando todo o sistema interligado nacional, composto por, aproximadamente, 3.700 barras, 5.300 elementos de transmissão e 65 GW de ponta de carga (2007).

\subsection{Seleção de Indisponibilidades}

As simulações relacionadas aos resultados apresentados nesta Seção consideram seis diferentes pedidos de desligamento para manutenção de equipamentos do sistema do Paraná. Esses casos foram estrategicamente selecionados do relatório técnico do ONS (ONS, 2006) por envolverem os desligamentos mais críticos do sistema de transmissão em estudo. Quando associados a outras contingências, tais desligamentos podem provocar cortes de carga. Estes pedidos de desligamento são definidos pelos seguintes casos:

Caso 1: Um TR 525/230 kV - 672 MVA - SE Curitiba.
Caso 2: Um TR 525/230 kV - 672 MVA - SE Londrina.

Caso 3: LT $500 \mathrm{kV}$ Cascavel Oeste - Salto Caxias.

Caso 4: LT 230 kV Campo Comprido - Pilarzinho.

Caso 5: LT 230 kV Uberaba - Umbará.

Caso 6: LT 230 kV Londrina(Copel) - Londrina (Eletrosul).

\subsection{Critério Probabilístico: Resultados mais Relevantes}

Seguindo a metodologia proposta na Seção 4, foram gerados seis novos casos base, um para cada indisponibilidade descrita pelos Casos 1 a 6 . Esses casos consideram, portanto, a condição $n$-1 a partir do caso base de carga média da Rede Básica de 2007. Embora as indisponibilidades definidas pelos casos 1-6 representem as piores condições para o sistema de transmissão que atende o Paraná, outras indisponibilidades citadas em (ONS, 2006) provocam também violações das condições de operação. Porém, essas são consideradas menos críticas, indicando que os casos selecionados permitem avaliar as situações mais severas para o sistema.

Utilizando o procedimento de confiabilidade padrão para o sistema de transmissão, foram determinados os índices de severidade de cada caso base (1-6). A Tabela 2 sumariza os resultados obtidos.

Essas análises foram realizadas através do uso do programa $\mathrm{NH} 2$, utilizando o método de simulação por enumeração de estados, conforme descrito no Passo 1 da metodologia proposta, e a base de dados estatística de (ONS-UFSC, 2006). Relembrando, essas análises levam em consideração: possibilidade de falhas em linhas de transmissão e transformadores de tensão primária igual ou superior a $230 \mathrm{kV}$ (124 elementos); limites de carregamento nominal e de emergência desses equipamentos, sempre considerando a configuração de 2007. Resultados adicionais e maiores detalhes referentes às análises de confiabilidade do sistema em análise podem ser encontradas em (Lima, 2007).

Os resultados da Tabela 2 apresentam, portanto, os índices de severidade obtidos para sete diferentes configurações do sistema, considerando o patamar de carga média de 2007.

O caso zero corresponde ao caso base original. A severidade obtida quando limites nominais de carregamento são considerados é da ordem de 7,5 minutos (segunda coluna). De acordo com a classificação do índice de severidade apresentada na Tabela 1, isso corresponde ao grau 1, o que sinaliza uma condição operativa do sistema de baixo risco. Caso sejam considerados os limites de emergência, o valor da severidade se reduz a aproximadamente 2,2 minutos (quarta coluna), que é ainda classificado como grau 1 . 
Tabela 2: Índice severidade, considerando limites nominais de carregamento e emergência, carga média/2007

\begin{tabular}{|c|c|c|c|c|}
\hline \multirow{2}{*}{ Caso } & \multicolumn{2}{|c|}{ Limites Nominais } & \multicolumn{2}{c|}{ Limite Emergência } \\
\cline { 2 - 5 } & S (minutos) & Diferença S (min.) & S (minutos) & Diferença S (min) \\
\hline $\mathbf{0 ~ ( 2 0 0 7 )}$ & 7,4871 & 0,0 & 2,1972 & 0,0 \\
\hline $\mathbf{1}$ & 20,6888 & 13,2017 & 2,2016 & 0,0044 \\
\hline $\mathbf{2}$ & 7,5028 & 0,0156 & 2,2032 & 0,0060 \\
\hline $\mathbf{3}$ & 7,4891 & 0,0020 & 2,1977 & 0,0005 \\
\hline $\mathbf{4}$ & 20,7497 & 13,2626 & 5,2877 & 3,0905 \\
\hline $\mathbf{5}$ & 7,7593 & 0,2721 & 2,3121 & 0,1149 \\
\hline $\mathbf{6}$ & 19,4763 & 11,9891 & 4,5895 & 2,3923 \\
\hline
\end{tabular}

Os casos 1 a 6 , mostrados na Tabela 2, levam em conta a indisponibilidade de um elemento, conforme descrito na Seção 5.2, gerando seis novos casos base de ordem $n-1$. Na Tabela 2, a coluna " $\mathrm{S}$ " indica o resultado da severidade total do sistema considerando o referido elemento removido. A coluna "Diferença $S$ " é a diferença entre o valor da severidade de cada caso com relação ao caso zero (caso base original). Essa coluna mostra, portanto, a contribuição na severidade do sistema devido à remoção do elemento em análise do caso base. Assim, de acordo com esses resultados, as indisponibilidades mais críticas são a da LT 230 kV Campo Comprido - Pilarzinho e do TR 525/230 kV da SE Curitiba, que acrescentam em torno de 13,2 minutos à severidade do sistema.

Portanto, se a metodologia de avaliação de risco probabilístico proposta na Seção 4 fosse adotada para avaliar os pedidos de desligamentos listados na Seção 5.1, e considerando os valores e diferenças de índice de severidade apresentados na Tabela 2, os seguintes desligamentos seriam aprovadas para realização durante o patamar de carga média diurna de dias úteis em 2007:

Caso 2: um TR 500/230 kV da SE Londrina;

Caso 3: LT 500 kV Cascavel Oeste - Salto Caxias;

Caso 5: LT 230 kV Uberaba - Umbará.

\subsection{Uso do critério determinístico}

Atualmente todas as solicitações de desligamento dos equipamentos definidos na Seção 5.1 são indeferidas pelo ONS para o patamar de carga média diurna de dias úteis. Ou seja, essas intervenções só podem ser realizadas em patamar de carga leve (domingos e feriados ou no período noturno). Esses resultados estão de acordo com os critérios determinísticos descritos na Seção 2, Figura 1, adotados atualmente pelo ONS.

\subsection{Avaliação dos resultados}

A Tabela 3 resume os resultados referentes à aprovação ou rejeição dos pedidos de desligamento para manutenção dos casos 1 a 6 em horário de carga média, considerando a adoção do critério determinístico atual (coluna 2) ou do critério probabilístico proposto (coluna 3).

Tabela 3: Resultados finais dos pedidos de desligamento para horário de carga média

\begin{tabular}{|c|c|c|}
\hline CASO & $\begin{array}{c}\text { Método } \\
\text { Determinístico }\end{array}$ & $\begin{array}{c}\text { Metodologia } \\
\text { Proposta }\end{array}$ \\
\hline $\mathbf{1}$ & Rejeitado & Rejeitado \\
\hline $\mathbf{2}$ & Rejeitado & APROVADO \\
\hline $\mathbf{3}$ & Rejeitado & APROVADO \\
\hline $\mathbf{4}$ & Rejeitado & Rejeitado \\
\hline $\mathbf{5}$ & Rejeitado & APROVADO \\
\hline $\mathbf{6}$ & Rejeitado & Rejeitado \\
\hline
\end{tabular}

Considerando que os casos selecionados (casos 1-6) são os mais críticos para o sistema do Paraná, pode-se concluir que a adoção do critério probabilístico proposto neste trabalho viabilizaria o atendimento de um grande número de pedidos de desligamento durante a carga média, sem expor o sistema a níveis de risco preocupantes (Lima, 2007).

Verifica-se ainda que realizar um ranking das contingências pode ser útil para o planejamento do sistema e para decisões acerca de pedidos de manutenção. No entanto, isso não pode ser realizado utilizando-se técnicas puramente determinísticas. A Tabela 2 mostra claramente que nem todas as contingências apresentam o mesmo impacto nos índices de risco do sistema. Notadamente, o critério determinístico não é capaz 
de fornecer esse tipo de informação, enfatizando a importância da metodologia proposta neste trabalho.

\section{CONCLUSÕES}

O presente artigo apresentou resultados inéditos relativos à análise de confiabilidade probabilística, utilizando o sistema elétrico que atende o Estado do Paraná como sistema teste. Os resultados obtidos corroboram com a proposta deste artigo de apresentar uma alternativa mais atraente e flexível para o planejamento da operação do que o atual critério $n$ 2 adotado pelo ONS. A proposta está fundamentada no nível de risco probabilístico que as intervenções programadas representam para o sistema elétrico.

A principal contribuição da nova metodologia é permitir que determinados desligamentos que hoje não são aprovados pelo ONS possam ser executados durante a carga média diurna de dias úteis e, ao mesmo tempo, não permitir que o sistema seja submetido a um nível de risco elevado. Acredita-se que a adoção do critério proposto permita estabelecer um equilíbrio entre as diferentes necessidades dos agentes do sistema e, principalmente, do consumidor.

Assim, espera-se que este artigo possa contribuir com a divulgação das potencialidades das técnicas probabilísticas, permitindo sua aplicação em ferramentas de auxilio à tomada de decisão. Adicionalmente, espera-se que os resultados obtidos com a aplicação da metodologia proposta em um sistema de potência real possam subsidiar discussões acerca deste tema, permitindo a quebra de paradigma relativa à adoção de critérios probabilísticos no planejamento da operação.

\section{AGRADECIMENTO}

Elizete Maria Lourenço agradece o apoio financeiro do CNPq, Conselho Nacional de Desenvolvimento Científico e Tecnológico.

\section{REFERÊNCIAS}

Billinton, R., Fotuhi-Firuzabad, M. and Bertling, L. (2001). Bibliography on the application of probability methods in power system reliability evaluation 1996-1999, IEEE Transactions on Power Systems 16(4): 595-602.

Cepel (2005). Programa nh2 - manual do usuário versão 7.3.

EPRI (1987). Composite system reliability evaluation methods, Technical report. EL-5178.

GCPS and SGC (1990). Gcps - grupo coordenador do planejamento do sistema; sgc - sub-grupo de confiabilidade. sistema computacional para análise de confiabilidade de sistemas de geração-transmissão de grande porte - projeto nh2 - metodologia de cálculo dos Índices de confiabilidade.

Lima, J. M. (2007). Uma análise minuciosa do risco probabilístico do sistema elétrico que atende o estado do paraná, Master's thesis, Departamento de Engenharia Elétrica - Universidade Federal do Paraná., Av: Maio, 2007.

URL: $\quad h t t p: / / d s p a c e . c 3 s l . u f p r . b r:$ 8080/dspace/bitstream/handle/

1884/10744/Uma \20An\EIIisel

20Minuciosal\%20dol\%20Riscol

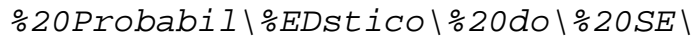

20quel20Aten.pdf; jsessionid=

E33D 75F9080786F73B7B4BD7870B6BFB?

sequence $=1$

Mello, J., Melo, A., Romero, S., Oliveira, G., Cunha, S., Morozowski, M., Pereira, M. and Fontoura, R. (1991). Development of a composite system reliability program for large hydrothermal power systems-issues and solutions, Third International Conference on Probabilistic Methods Applied to Electric Power Systems, 1991., pp. 64-69.

Negreiros, A., M, G. L. F., Torres, G. L., Schilling, M. T., Souza, J. C. S. and Couto Filho, M. B. (2007). Avaliação do risco probabilistico da rede básica do sin utilizando dados estocásticos realistas, XIX SNPTEE, Rio de Janeiro.

ONS (2004). Procedimentos e critérios para análise de confiabilidade, Technical report. ONS 2.1-131/2004 version 15.07 .

ONS (2006). Planejamento elétrico da operação para o sistema interligado nacional, Technical report. ONS RE3/365/2006.

ONS-UFSC (2006). Indicadores de desempenho probabilístico de componentes de geração e transmissão do sin. desenvolvimento de uma base de dados aplicada a estudos de confiabilidade para sistemas de geração e transmissão, Technical report. Volume I.

URL: http://www.ons.org.br/download/ ampliacaol_reforcos/confiabilidade/ volume $\$ \%20i-indicadores \_desempenhol _revisado.pdf

Ran, M. (2003). Deterministic/probabilistic evaluation in composite system planning, Master's thesis, College of Graduate Students and Research, Department of Electrical Engineering University of Saskatchewan Saskatoon.

Rei, A., Schilling, M. and Melo, A. (2006). Monte carlo simulation and contingency enumeration in bulk power 
systems reliability assessment, International Conference on Probabilistic Methods Applied to Power Systems, 2006. PMAPS 2006., pp. 1-6.

Schilling, M., Filho, M. D. C., da Silva, A. L., Billinton, R. and Allan, R. (1995). An integrated approach to power system reliability assessment, International Journal of Electrical Power and Energy Systems 17(6): 381-390.

Schilling, M. T., Billinton, R. and dos Santos, M. G. (2009). Bibliography on power systems probabilistic security analysis 1968-2008, International Journal of Emerging Electric Power Systems 10(3): 1-48.
Schilling, M. T., Soares, N. H. M. and Luz, J. C. F. (1998). Discernimento de indicadores de risco, Revista Eletroevolução (13): 30-88.

Schneider, A.W., J., Raksany, J., Gunderson, R., Fong, C., Billington, R., O'Neill, P. and Silverstein, B. (1989). Bulk system reliability-measurement and indices, IEEE Transactions on Power Systems 4(3): 829-835.

Soares, N. H. M. (2002). Risco probabilístico de referência do sistema elétrico brasileiro, Master's thesis, Universidade Federal de Itajubá. 